\title{
Slow fluctuations in enhanced Raman scattering and surface roughness relaxation
}

\author{
D. B. Lukatsky ${ }^{a *}$, G. $\operatorname{Haran}^{b}$, and S. A. Safran ${ }^{a}$ \\ ${ }^{a}$ Department of Materials and Interfaces, and ${ }^{b}$ Department of Chemical Physics, \\ Weizmann Institute of Science, 76100 Rehovot, Israel
}

\begin{abstract}
We propose an explanation for the recently measured slow fluctuations and "blinking" in the surface enhanced Raman scattering (SERS) spectrum of single molecules adsorbed on a silver colloidal particle. We suggest that these fluctuations may be related to the dynamic relaxation of the surface roughness on the nanometer scale and show that there are two classes of roughness with qualitatively different dynamics. The predictions agree with measurements of surface roughness relaxation. Using a theoretical model for the kinetics of surface roughness relaxation in the presence of charges and optical electrical fields, we predict that the high-frequency electromagnetic field increases both the effective surface tension and the surface diffusion constant and thus accelerates the surface smoothing kinetics and time scale of the Raman fluctuations in manner that is linear with the laser power intensity, while the addition of salt retards the surface relaxation kinetics and increases the time scale of the fluctuations. These predictions are in qualitative agreement with the Raman experiments.
\end{abstract}

In a recent experiment by Weiss and Haran [1], large spectral fluctuations in the relative intensities of different Raman lines that varied on a time scale of a few tens of seconds, were measured in the surface-enhanced Raman scattering (SERS) of single rhodamine 6G molecules adsorbed on silver nanocrystals. The rate of spectral fluctuations was demonstrated to increase with laser intensity and decrease with addition of salt in the solution of the silver colloidal particles. In addition, a decay of the overall intensity of the scattering was observed on a scale of the order of hundreds of seconds; this decay was also correlated with the laser intensity [1]. Finally, fluctuations of the overall intensity on a time scale comparable with that of the fluctuations of the individual spectral lines were measured in Refs. [1]. These fluctuations, sometimes termed "blinking", are frequently observed in single-molecule SERS studies [2-5]. The long time scale observed in the modulations of the spectrum of molecules adsorbed on silver colloidal particles, was suggested [1] to arise from (slow) motion of the adsorbed molecule, which leads to variation of a charge-transfer interaction between the molecule and the surface.

In this paper, we propose an alternative interpretation of both the slow SERS spectrum fluctuations, and of the decay and the fluctuations of the overall intensity ("blinking") of the SERS spectrum measured in Ref. [1] and show that the time scales for these phenomena are consistent with the relaxation of the surface roughness on the nanometer scale [6]. It is well known that a significant part of the enhancement of the SERS signal is due to the surface roughness $[7,8]$. Using theoretical models for the kinetics of surface roughness relaxation in the presence of charges and optical electrical fields, we predict the dependence of the time scale of the fluctuations on the laser power intensity and on the amount of added salt in agreement with the observations.

Conventionally, the SERS enhancement mechanism is separated into the electromagnetic (EM) field enhancement and the chemical enhancement [3]. The relaxation of surface roughness can affect both the EM and chemical mechanism of the SERS enhancement, depending on the characteristic length-scale and the amplitude of the relaxation modes. The surface relaxation dynamics itself, however, is governed by the EM mechanism in our model, as we elaborate below. These constitute the starting point of our analysis.

Our dynamical model applies both to equilibrium fluctuations and to the decay to equilibrium (a smooth surface) of non-equilibrium, surface roughness that can have large amplitudes, depending on the initial preparation of the nanoparticles. The amplitude of equilibrium fluctuations is typically small for perfectly smooth surfaces [9]. However, rough surfaces (where the roughness due to sample preparation decays slowly) show larger thermal fluctuations [10]. Thus, the roughness fluctuations may have a strong effect on the SERS enhancement mechanism and may lead to the observed fluctuations in the relative intensities of the different Raman lines.

We first estimate the time scale for relaxation of the surface roughness. We use a model for surface relaxation developed by Mullins [11] based on an isotropic expression for the surface energy. This theory is applicable either above the roughening transition temperature or for vicinal surfaces (that are not perfectly smooth on the

*Present address: FOM Institute for Atomic and Molecular Physics, Kruislaan 407, 1098 SJ Amsterdam, The Netherlands 
atomic scale), even below the roughening temperature. The dynamics of ideal, high symmetry surfaces involves the creation of steps and facets [12] and is outside the scope of our work. Assuming that surface diffusion (with diffusion constant $D_{s}$ for single atom motion on the surface) is the only relevant process, leads one to the kinetic equation:

$$
\frac{\partial h}{\partial t}=-\Gamma \nabla^{4} h
$$

where $h(\vec{\rho}, t)$ is the local height, $\gamma_{0}$ is the surface tension, $\vec{\rho}=(x, y)$ is the in-plane position vector and $\vec{\nabla}=$ $\left(\partial_{x}, \partial_{y}\right)$, and $\Gamma=D_{s} a^{4} \gamma_{0} / k_{B} T$ with $a$ being an atomic length scale (e.g. the nearest-neighbor distance, for silver $a \simeq 2.8 \AA$ ). To estimate the characteristic time of the SERS spectral fluctuations, we analyze the height-height dynamic correlation function, $\mathcal{C}_{h h}(\vec{\rho}, t) \equiv\langle h(\vec{\rho}, t) h(0,0)\rangle$.

$\mathcal{C}_{h h}(\vec{\rho}, t)$ can be straightforwardly obtained from Eq. (1) (see e.g., Refs. $[9,13]$ ) assuming that the surface atoms are in contact with a thermal bath: $\mathcal{C}_{h h}(\vec{\rho}, t)=$ $\frac{k_{B} T}{2 \pi \gamma_{0}} \int_{q_{0}}^{\infty} \frac{e^{-\Gamma q^{4} t}}{q} J_{0}(q \rho) \mathrm{d} q$, where $J_{0}(x)$ is the Bessel function, and $q_{0}=2 \pi / L$, with $L$ being the system size. To estimate the characteristic relaxation time, $\tau$, numerically, one can adopt the criterion: $\mathcal{C}_{h h}\left(\vec{\rho}_{0}, \tau\right) / \mathcal{C}_{h h}\left(\vec{\rho}_{0}, 0\right)=$ $1 / \exp (1)$, were $\rho_{0}$ is of the order of a size of the adsorbed molecule.

An accurate estimate of the relaxation time, $\tau$, requires a knowledge of the surface diffusion constant, $D_{s}$, of silver estimated in the range $D_{s} \simeq 1.8 \times 10^{-15} \mathrm{~cm}^{2} / \mathrm{sec}$ [14] to $D_{s} \sim 10^{-14} \mathrm{~cm}^{2} / \mathrm{sec} \quad[15]$. Using $L \simeq 50 \mathrm{~nm}$ of the order of a size of silver colloids in the experiment [1], surface tension of $\mathrm{Ag}$ [16], $\gamma_{0} \simeq 1500 \mathrm{erg} / \mathrm{cm}^{2}, \rho_{0} \simeq 6$ $\AA$, we find that the correlation time of the equilibrium fluctuations is $\tau \simeq 18 \mathrm{sec}$. This shows that the time scale for the equilibrium relaxation of surface roughness on nanometer scale at room temperature is comparable to the correlation times of the slow fluctuations of the SERS spectrum measured in [1]. An analytic estimate for the correlation time can be obtained from the asymptotic long-time form: $\mathcal{C}_{h h}\left(\vec{\rho}_{0}, t\right) \simeq \mathcal{C}_{h h}(0, t) \sim \frac{k_{B} T}{\gamma_{0}} \frac{\exp (-t / \tau)}{t / \tau}$, where $\tau=1 / \Gamma q_{0}^{4}$. We emphasize that the relaxation time, $\tau$, is sensitive to the numerical values used for the lattice constant as well as to the wavelength associated with the size of a colloid.

In addition to the small amplitude, equilibrium fluctuations of the surface, much larger amplitude surface roughness may arise due to the sample preparation. These features tend to decay in time, leading to a smooth surface at the atomic level and this relaxation to equilibrium of a relatively smooth surface (and hence less enhancement of the Raman scattering), leads to the overall decay of the SERS spectrum intensity. The loss of the Raman signal is a well known phenomenon that also occurs in electrochemical $[17,18]$ and ultrahigh vacuum $[19,20]$ SERS systems. This effect was attributed to a diffusive loss of surface adatoms in a number of investigations (see e.g., introduction in Refs. [17,18]); however, none of these, to the best of our knowledge, explain the slow time scale in terms of cooperative surface tension effects nor do they discuss the dependence of the decay on the laser optical field and on added salt.

Another type of experiment to which our kinetic model is relevant, directly measures the dynamics of artificially created, nanoscale surface roughness features [15]. Since the surface profiles in the experimental systems are, in general, far from being simple sinusoids, we predict the relaxation time for two important classes of surface features.

To estimate the relaxation time for the two classes of surface features we consider two specific surface roughness profiles at an initial time $t=0$ : (i) a non-massconserving profile, modelled as a Gaussian protrusion where $h>0, h(\vec{\rho}, t=0)=h_{0} e^{-\alpha \rho^{2}}$ and (ii) a massconserving profile, modelled as a region where a protrusion with $h>0$ is adjacent to an indentation where $h<0$. The average value of $h$ over the entire surface is zero and for convenience we consider: $h(\vec{\rho}, t=0)=$ $h_{0} e^{-\alpha \rho^{2}}\left(1-\alpha \rho^{2}\right)$, where $\alpha^{-1 / 2}$ is the characteristic lateral scale of the feature, and $h_{0}$ is the amplitude. The experiments of Ref. [15] correspond to the mass-conserving case (ii) (possibly because of the manner in which the surface was scratched), while we expect that general surface roughness of colloidal particles, applicable to the Raman experiments, to be more similar to the non-massconserving case (i). It is straightforward to compute the time evolution of these two types of profiles, by solving Eq. (1) with the corresponding initial conditions of cases (i) and (ii). The time dependence of the decay of the maximum height (located at the origin, $\rho=0$ ) of these Gaussian-like peaks can be obtained analytically. For case (i), we find $h(0, t)=h_{0}\left(\pi \tau_{g} / t\right)^{1 / 2} \beta\left(\tau_{g} / t\right)$. For case (ii) we find $h(0, t)=h_{0}\left[2 \tau_{g} / t-2 \sqrt{\pi}\left(\tau_{g} / t\right)^{3 / 2} \beta\left(\tau_{g} / t\right)\right]$, where $\beta(x)=\exp (x) \operatorname{erfc}(\sqrt{x})$ and $\tau_{g}=k_{B} T /\left(64 \gamma D_{s} \alpha^{2} a^{4}\right)$ is the characteristic decay time, and $\operatorname{erfc}(x)$ is the complementary error function.

The most important observation is that these two types of profiles have qualitatively different smoothing kinetics - the mass conserving profile (ii) decays much faster since in this case the transport of matter need only occur near the boundary between the protrusion and the indentation; that is, atoms are locally transferred from the region where $h>0$ to the region where $h<0$. For case (i) of the non-mass-conserving profile, the matter must be transported to a much larger scale. This is reflected in the expressions for the asymptotic, long time $\left(\tau_{g} \ll t\right)$ evolution of the height. For case (i), the decay of the maximum of the profile (located at the origin), $h(0, t) \simeq h_{0} \pi^{1 / 2}\left(\tau_{g} / t\right)^{1 / 2}$. For case (ii) $h(0, t) \simeq 2 h_{0} \tau_{g} / t$ tends to zero much faster.

We now use this model for surface smoothing in case 
(ii) to estimate the decay time for the (approximately) mass-conserving surface features studied in Ref. [15]. Using their estimate for the diffusion constant at zero external potential, $D_{s} \simeq 10^{-14} \mathrm{~cm}^{2} / \mathrm{sec}$ and a value for $\alpha^{-1 / 2} \simeq 8 \mathrm{~nm}$ that corresponds to the extent of the scratch, we obtain that a mass conserving, Gaussian profile with initial amplitude $h_{0} \simeq 2 \mathrm{~nm}$ decays to an atomicscale estimated as $h(\tau) \simeq 0.3 \mathrm{~nm}$, in a time $\tau \simeq 200 \mathrm{sec}$. This theoretical estimate is consistent with the experimentally measured times in the scratch experiments of Hirai [15]. An estimate of the decay time for case (i) (i.e., the non-mass-conserving profile), yields for the same parameters, a decay time that is about 15 times slower, $\tau \simeq 3100 \mathrm{sec}$. In the case of colloidal particles, we expect that typical, non-equilibrium features (preparation dependent roughness) may be non-mass-conserving protrusions or indentations and that the kinetics of case (i) would apply. We estimate, for instance, that a more localized, Gaussian protrusion of amplitude $1 \mathrm{~nm}$ and extent $\alpha^{-1 / 2} \simeq 6 \mathrm{~nm}$ would decay to an atomic size of 0.3 $\mathrm{nm}$ in a time $200 \mathrm{sec}$, consistent with the times measured in the Raman experiments [1].

The Raman experiments show a systematic dependence of the modulation and relaxation time scales on the salt and the electric field as mentioned at the beginning. Since the characteristic time scales vary inversely with the product of the surface tension and diffusion constant (see Eq. (1)), we consider: (i) the effect of salt on the surface tension (ii) the effect of the laser field on the time scale via its effect on the surface tension (iii) the effect of the laser field on the surface diffusion constant. To treat the effects of surface charges [21] and the laser electric field on the silver surface tension and hence on the surface relaxation, we model the charged colloidal interface as an elastic, almost planar surface, with a fixed and uniform surface density of charge $\sigma$, and height $h(\vec{\rho})$. The surface tension, given by calculating the free energy cost (including the electrostatic effects due to the charges and the salt) of deviations of the surface from the planar geometry [22] is: $\gamma_{e}=\frac{2 \pi \sigma^{2}}{\epsilon \kappa}=\frac{\epsilon E_{s}^{2}}{8 \pi \kappa}$, where $\epsilon \simeq 80$ is the dielectric constant of water, $\kappa^{-1}$ is the Debye screening length, $\kappa^{2}=8 \pi \ell_{B} \mathcal{Z}^{2} n$, here $\ell_{B}=\frac{e^{2}}{\epsilon k_{B} T} \simeq 7 \AA$ is the valence of salt ions, and $n$ is the salt concentration, $E_{s}=4 \pi \sigma / \epsilon$ is the electric field at the colloid surface. This result can also be obtained from a scaling argument: the tension is the product of the energy density and the volume divided by the cross-sectional area; this is proportional to energy density $\epsilon E^{2}$ multiplied by a characterisitic length, which here is the Debye length, $\kappa^{-1}$.

This result shows that with the addition of salt, the effective surface tension decreases, $\gamma_{e} \sim n^{-1 / 2}$. This leads to an increase of the relaxation time, $\tau$, as observed. Taking the experimental values of the parameters used above, we see that in order to obtain an effect of order unity (i.e., an effective electrostatic surface tension, $\gamma_{e}$, equal to the bare surface tension of silver, $\gamma_{e}=\gamma_{0} \simeq 1500 \mathrm{erg} / \mathrm{cm}^{2}$ ) the surface of a silver colloidal particle must have a surface charge density, $\sigma \simeq 10 \mathrm{e} / \mathrm{nm}^{2}$ in $100 \mathrm{mM}$ salt solution. This is a rather high surface charge density, but is still within a realistic range for the experiments.

To quantify the contribution of the laser field to the surface energy and diffusion constant of a silver nanocrystal requires an accurate value for the enhanced surface electric field; this requires an accurate model of the microscopic mechanism of the enhancement, which is not yet completely understood [7]. However, we can predict the functional dependence of the both the surface tension as well as the surface diffusion constant on the field at the surface. The surface energy density, $u$, of a semi infinite metal sample with a planar surface boundary in the presence of a high-frequency, optical electromagnetic field, is [23]:

$$
u(z)=\frac{1}{8 \pi}\left[\frac{\partial(\omega \varepsilon(\omega))}{\partial \omega}|\vec{E}(z)|^{2}+\frac{\partial(\omega \mu(\omega))}{\partial \omega}|\vec{H}(z)|^{2}\right],
$$

where $\vec{E}(z)=\vec{E}_{0} e^{i k z}$ is the spatially-dependent part of the electric field vector with amplitude, $\vec{E}_{0}$, and the plane, $z=0$, corresponds to the metal interface plane; the same definitions apply to the magnetic field, $\vec{H}$. For the frequency-dependent dielectric function, $\varepsilon(\omega)$, we use the simplest dispersion model of the free, classical electron gas [23]: $\varepsilon(\omega)=1-\left(\frac{\omega_{p}}{\omega}\right)^{2}$, where $\omega_{p} \simeq 9.2$ $\mathrm{eV} \simeq 1.4 \times 10^{16} \mathrm{sec}^{-1}$ is the plasma frequency of silver, and $\omega \simeq 3.5 \times 10^{15} \mathrm{sec}^{-1}$ for the $530 \mathrm{~nm}$ wavelength laser beam. For the optical frequencies relevant to the experiments [1], $\mu(\omega)=1$. Using the dispersion relation [23], $k^{2}=\frac{\varepsilon(\omega)}{c^{2}} \omega^{2}$, we obtain: $k \equiv i \chi=i \frac{\sqrt{\omega_{p}^{2}-\omega^{2}}}{c}$, and therefore, both the electric and magnetic fields decay exponentially within the silver colloidal particle with a typical decay length [24], $\chi^{-1}$, of $22 \mathrm{~nm}$. In this frequency region therefore, the metal is reflecting with a skin depth, $\chi^{-1}$. Using the relationship between $\vec{H}$ and $\vec{E}$ [23], we obtain the time-averaged energy per unit surface area (i.e., effective surface tension), $\gamma_{e m}=\int_{0}^{\infty} u(z) d z$, in the presence of an electromagnetic field: $\gamma_{e m}=\frac{E_{0}^{2}}{8 \pi \chi}$. Again, this can be predicted by a scaling argument: the tension is the product of the energy density (proportional to the laser intensity) and the characteristic length, here the skin depth, in qualitative agreement with the experiment.

However, an estimate of the magnitude of this effect using the bare values $E_{0} \simeq 0.6\left(\mathrm{erg}^{1 / 2} / \mathrm{cm}^{3 / 2}\right) \simeq 180 \mathrm{~V} / \mathrm{cm}$, (corresponding to a laser power density of $100 \mathrm{~W} / \mathrm{cm}^{2}$ ) and $\chi^{-1} \simeq 22 \mathrm{~nm}$ is $\gamma_{e m} \simeq 3 \times 10^{-8} \frac{\mathrm{erg}}{\mathrm{cm}^{2}}$. This quantity, $\gamma_{e m}$, is thus about $10^{11}$ times smaller than the bare coefficient of surface tension of silver, $\gamma_{0}$ ! It is known that the electric field at the surface in the case of SERS is greatly enhanced by the surface plasmon resonances in 
the system $[7,25]$. Recent experiments $[2,3]$ report that the SERS enhancement factor, (theoretically predicted to be proportional to $\left.\left(\mathcal{E} / E_{0}\right)^{4}\right)$ where $\mathcal{E}$ is the intensity of scattered, enhanced optical field (see also Refs. $[27,28]$ )), reaches a value of $10^{14}-10^{15}$. This enhancement is still not large enough to bridge the gap between our prediction for the optical field-induced effective surface tension, $\gamma_{e m}$, and the bare surface tension of silver, $\gamma_{0}$. We note, however, that the intensity of the localized field at the surface of a metal colloid may exceed the intensity of the radiated, enhanced optical field [7]. Atomic scale, globally distributed roughness (adatoms, terraces, kinks, small islands) may produce an extra enhancement of the electric field at the surface of a colloidal particle, as well, although a quantitaive understanding of this mechanism is still lacking (see e.g., [26]). In summary, the scaling of the tension and hence the time scale for surface relaxation with the electric field is qualitatively correct; however, the magnitude depends on the actual value of the surface field.

Although the effect of the field on the surface tension may turn out to be too small to matter, the change in the local diffusion constant of silver adatoms with field may be more significant; the diffusion constant enters the kinetic equations discussed above. Indeed, in the case of a static electric field applied to a roughened metal interface, Hirai et al. [15] show from their experiment that the surface diffusion coefficient, $D_{s}$, depends exponentially on the applied potential difference. This is in accord with theoretical calculations [29] of surface diffusion in the presence of external fields, in the limit where the electrostatic energy is much larger than the thermal energy. This is the case in the static experiments where the potential drop occurs on a length scale of $\sim 1 \mathrm{~nm}$ of order of the Debye length; this gives rise to a very large electric field. However, this limit is not applicable to the Raman experiments. The electrolyte cannot respond to the high frequency optical field and provides no screening of the laser field. The potential drops over a length scale given by the optical wavelength, and not the Debye length. The resulting field is thus orders of magnitude smaller than in the static case. Indeed, the Raman experiments show only a linear dependence of the rate of the SERS spectral fluctuations on the laser intensity and no exponential behavior.

Calculations [29] of surface diffusion in the presence of external fields in the limit where the field energy is smaller than the thermal energy, show that the surface diffusion coefficient depends linearly on the laser intensity [30]: $D_{s} \simeq D_{s}\left(E_{0}=0\right)\left(1+W^{2}\right)$ where $W \equiv$ $e E_{0} a /\left(4 k_{B} T\right) \ll 1$. This trend agrees with the experimental observation that the time scale varies with the laser intensity. However, as in the case of the surface tension, this requires a significant enhancement of the local, surface field to yield a measureable effect. We note that even if the field contribution to the tension is small, the effect on the diffusion constant may be significantly larger since the field dependence of the tension depends on the ratio of the intensity to the bare silver surface tension while the correction to the diffusion constant varies with the ratio of the intensity to the thermal energy, which is about two orders of magnitude smaller than the energy associated with the surface tension of silver.

The best way to verify our predictions would be to perform SERS measurements on surfaces with in situ control of surface roughness by e.g., the methods described in Ref. [15]. Monitoring the SERS spectrum as a function of the surface roughness, the salt concentration, and the laser intensity should provide an ultimate test of our idea.

We thank J. Imry, D. Kandel, and A. Nitzan for useful discussions. SAS acknowledges the support of the US Israel Binational Science Foundation and the Schmidt Minerva Center. GH is the incumbent of the Benjamin H. Swig nad Jack D. Weiler career development chair.

[1] A. Weiss and G. Haran, J. Phys. Chem. B, 105, 12348 (2001).

[2] S. Nie and S. R. Emory, Science, 275, 1102 (1997).

[3] W. E. Doering and S. Nie, J. Phys. Chem. B, 106, 311 (2002).

[4] A. M. Michaels, M. Nirmal, and L. E. Brus, J. Am. Chem. Soc. 121, 9932 (1999).

[5] E. J. Bjerneld, P. Johansson, and M. Kall, Single Mol., 1, 239 (2000).

[6] We note that in the single-molecule SERS experiments in addition to intensity fluctuations, frequency fluctuations are usually observed (see e.g., Ref. [3]). These fluctuations were much less pronounced compared with the intensity fluctuations in Ref. [1]. These large, intensity fluctuations are the focus of our work.

[7] M. Moskovits, Rev. Mod. Phys., 57, 783 (1985).

[8] A. Otto et al., Surf. Sci., 138, 319 (1983).

[9] S. A. Safran, Statistical Thermodynamics of Surfaces, Interfaces, and Membranes (Addison-Wesley Publishing Com., Reading, MA 1994).

[10] One can see this by expanding the full expression for the surface energy, $\gamma \int \mathrm{d} \vec{\rho}\left[1+(\nabla h)^{2}\right]^{1 / 2}$, to second order about the average roughness and calculating the amplitude of thermal fluctuations.

[11] W. W. Mullins, J. Appl. Phys., 57, 333 (1957).

[12] N. Israeli and D. Kandel, Phys. Rev. Lett. 88, 116103 (2002).

[13] P. M. Chaikin and T. C. Lubensky, Principles of condensed matter physics (Cambridge University Press, Cambridge, 1995) Ch. 7.

[14] W. W. Pai et al., Phys. Rev. Lett., 79, 3210 (1997).

[15] N. Hirai, H. Tanaka, S. Hara, Appl. Surf. Sci., 130-132, 506 (1998). 
[16] M . J. Mehl and D. A. Papaconstantopoulos, Phys. Rev. B, 54, 4519 (1996).

[17] Y.-S. Choi, J.-J. Kim, and S. Miyajima, Chem. Phys. Lett., 255, 45 (1996) and refernces therein.

[18] L. A. Dick et al., J. Phys. Chem. B, 106, 853 (2002).

[19] U. Erturk, I. Pockrand, and A. Otto, Surf. Sci., 131, 367 (1983).

[20] C. Douketis et al., J. Chem. Phys., 113, 11315 (2000).

[21] The colloid surface is charged due to the presence of citrate ions in the solution.

[22] R. E. Goldstein, A. I. Pesci, and V. Romeo-Rochin, Phys. Rev. A, 41, 5504 (1990).

[23] L. D. Landau and E. M. Lifshitz, Electrodynamics of continuous media, (Pergamon Press, Oxford 1984).

[24] U. Kreibig and M. Vollmer, Optical Properties of Metal Clusters, (Springer-Verlag, Berlin, 1995).
[25] D. J. Bergman and A. Nitzan, Chem. Phys. Lett., 88, 409 (1982).

[26] A. Otto, Phys. Stat. Sol., 188, 1455 (2001).

[27] H. X. Xu et al., Phys. Rev. E, 62, 4318 (2000).

[28] S. Corni and J. Tomasi, J. Chem. Phys., 116, 1156 (2002).

[29] J. Kallunki, M. Dube, and T. Ala-Nissila, Surf. Sci., 460, 39 (2000).

[30] This result was derived under the assumption that the laser frequency is smaller than the frequency of the thermal noise. The thermal noise arising from phonons has a typical frequency of $10^{13} \mathrm{sec}^{-1}$ which is about two orders of magnitude smaller than the laser frequency. However, very high frequency thermal noise may arise from the coupling of the silver ions to the surface plasmons. 